PROCEEDINGS OF THE

AMERICAN MATHEMATICAL SOCIETY

Volume 139, Number 6, June 2011, Pages 1939-1953

S 0002-9939(2011)10856-6

Article electronically published on January 6, 2011

\title{
RAMANUJAN BIGRAPHS ASSOCIATED WITH $S U(3)$ OVER A $p$-ADIC FIELD
}

\author{
CRISTINA BALLANTINE AND DAN CIUBOTARU
}

(Communicated by Matthew A. Papanikolas)

\begin{abstract}
We use the representation theory of the quasisplit form $G$ of $S U(3)$ over a $p$-adic field to investigate whether certain quotients of the Bruhat-Tits tree associated to this form are Ramanujan bigraphs. We show that a quotient of the tree associated with $G$ (which is a biregular bigraph) is Ramanujan if and only if $G$ satisfies a Ramanujan type conjecture. This result is analogous to the seminal case of $P G L_{2}\left(\mathbb{Q}_{p}\right)$ considered by Lubotzky, Phillips, and Sarnak. As a consequence, the classification of the automorphic spectrum of the unitary group in three variables by Rogawski implies the existence of certain infinite families of Ramanujan bigraphs.
\end{abstract}

\section{INTRODUCTION}

This paper is concerned with the question of constructing infinite families of Ramanujan bigraphs, i.e., biregular, bipartite graphs with certain strong connectivity conditions. Following the classical case of regular graphs, the condition is that the second largest eigenvalue of the adjacency matrix is asymptotically as small as possible. The notion of a Ramanujan bigraph is made precise in Definition 2.4. and it is motivated by the result of [9] (see Proposition 2.3), which is the analogue for bigraphs of the well-known bound of Alon-Boppana for regular graphs. There is also an important notion of a weak Ramanujan bigraph which has to do with the multiplicity of the eigenvalue 0 in the adjacency matrix of the bigraph. Every Ramanujan bigraph is immediately weak Ramanujan.

Following the approach of [15], where infinite families of regular Ramanujan graphs were contructed using the representation theory of $P G L\left(2, \mathbb{Q}_{p}\right)$ and deep results from the theory of automorphic forms, we construct families of Ramanujan bigraphs using the quasisplit form $G$ of the group $S U(3)$ defined over a $p$-adic field $K$. For the group $G$, the Bruhat-Tits tree $\tilde{X}$ is an infinite biregular bipartite bigraph with valencies $q^{3}+1$ and $q+1$, where $q$ is the order of the residue field of $K$. Let $I$ denote the stabilizer in $G$ of an edge of $\tilde{X}$. This is an Iwahori subgroup of $G$. Assume that we have a discrete co-compact subgroup $\Gamma$ of $G$ which acts without fixed points. Our first result (Theorem 4.2) shows that the quotient tree $X=\tilde{X} / \Gamma$ is a Ramanujan bigraph if and only if a certain Ramanujan type conjecture is satified. More precisely, we have:

Received by the editors June 1, 2010.

2010 Mathematics Subject Classification. Primary 11F70, 22E50.

The second author was supported in part by NSA-AMS 081022 .

(C)2011 American Mathematical Society Reverts to public domain 28 years from publication 
Theorem 1.1. The quotient tree $X=\tilde{X} / \Gamma$ is a Ramanujan bigraph if and only if every nontrivial irreducible unitary representation of $G$ with Iwahori fixed vectors that appears in $L^{2}(G / \Gamma)$ is tempered.

To establish this equivalence, we make use of the Iwahori-Hecke algebra $\mathcal{H}(G, I)$, its representation theory (see $\S 3$ ) and its action on $X$ via adjacency operators (see $\S 4$, following [10]).

In order to use this criterion to show the existence of families of Ramanujan bigraphs, we rely on the results of [19, where the classification of the automorphic spectrum of $U(3)$ is completed. Let $\mathbb{G}$ be an inner form of $S U(3)$ defined over a global field such that at a place $v$, the group $\mathbb{G}_{v}$ of points over the local field is isomorphic to $G$. The group $\mathbb{G}$ is constructed from a central simple algebra $D$ of degree 3 over a quadratic extension $E$ of the global field by means of an involution $\alpha$ of the second kind. We construct an infinite family of discrete co-compact subgroups $\Gamma_{i, v, n}$ of $G$ ( $i$ ranges over a finite set, $n$ over an infinite set; see 4.3 ) and consider the family of quotient trees $X_{i, v, n}=\tilde{X} / \Gamma_{i, v, n}$. Translating the results of [19] in this setting, one finds the following result.

Theorem 1.2. Assume that at the infinite place, $\mathbb{G}_{\infty}$ is compact. Then $X_{i, v, n}$ is a Ramanujan bigraph if and only if it is a weak Ramanujan bigraph. If one assumes that the central simple algebra $D$ (which gives rise to $\mathbb{G}$ ) is not split over $E$, then the graphs $X_{i, v, n}$ are Ramanujan bigraphs.

The paper is organized as follows. In $\S 2$, we recall the basic definitions about Ramanujan graphs and bigraphs and the associated zeta functions. In $\S 3$, we introduce the Iwahori-Hecke algebra of the quasisplit $S U(3)$ and present the classification of its modules, including the unitary dual. In $\S 4$, we explain how the spectral theory of the Iwahori-Hecke algebra is related to the eigenvalues of the zeta function of the Bruhat-Tits bigraph and obtain the main results listed above.

\section{Preliminaries}

2.1. As motivation, we first recall briefly the notion of a regular Ramanujan graph. For any $k$-regular graph $X$, we denote by $V(X)$ its vertex set and by $E(X)$ its edge set. We denote by $\operatorname{Ad}(X)$ the adjacency matrix of $X$ and by $\operatorname{Spec}(X)$ the spectrum of $X$, i.e., the collection of eigenvalues of $\operatorname{Ad}(X)$. Notice that we have $k \in \operatorname{Spec}(X)$. Denote by

$$
\lambda(X)=\max \{|\lambda|: \lambda \in \operatorname{Spec}(X),|\lambda| \neq k\}
$$

the second largest eigenvalue of $\operatorname{Ad}(X)$ in absolute value. If $A$ is a collection of vertices of $G$, the boundary of $A$, denoted $\partial A$, is given by

$$
\partial A=\{x \in V(X) \backslash A:\{x, y\} \in E(X) \text {, for some } y \in A\} .
$$

Definition 2.1. A finite regular graph $X$ on $n$ vertices and of degree $k$ is called an $(n, k, c)$-expander if for every subset $A$ of $V(X)$ with $|A| \leq \frac{n}{2}$ we have $|\partial A| \geq c|A|$. The constant $c$ is called the expansion coefficient.

The expansion coefficient of a regular graph $X$ depends on $\lambda(X)$. More precisely, it is known ([15]) that a finite $k$-regular graph $X$ on $n$ vertices is an $(n, k, c)$ expander with $2 c=1-\lambda(X) / k$. Thus, good expanders have small $\lambda(X)$. However, asymptotically $\lambda(X)$ cannot be made arbitrarily small, as known from the classical 
result of Alon-Boppana, which shows that if $X_{n, k}$ is a $k$-regular graph on $n$ vertices, then

$$
\liminf _{n \rightarrow \infty} \lambda\left(X_{n, k}\right) \geq 2 \sqrt{k-1}
$$

This bound leads to the following natural definition.

Definition $2.2([15)$. A $(q+1)$-regular graph $X$ is called a Ramanujan graph if $\lambda(X) \leq 2 \sqrt{q}$.

Infinite families of Ramanujan graphs of constant degree have been constructed using the Bruhat-Tits tree for $P G L\left(2, \mathbb{Q}_{p}\right)$ and deep results from number theory [15. 18. We will be interested in the analogous notion and constructions for a Ramanujan bigraph.

2.2. Recall that a $(k, l)$-biregular bigraph $X$ is a bipartite graph in which all vertices of one color have degree $k$ and all vertices of the other color have degree $l$. If $X$ is a $(k, l)$-biregular bigraph, the trivial eigenvalues of its adjacency matrix are $\pm \sqrt{k l}$. As before, let

$$
\lambda(X)=\max \{|\lambda|: \lambda \in \operatorname{Spec}(X),|\lambda| \neq \sqrt{k l}\}
$$

denote the absolute value of the second largest eigenvalue, in absolute value, of $X$. Then, analogous to the Alon-Boppana bound we have the following result due to Feng and Li $[9]$.

Proposition $2.3([9])$. If $X_{k, l, n}$ is a $(k, l)$-biregular bigraph on $n$ vertices, then

$$
\liminf _{n \rightarrow \infty} \lambda\left(X_{k, l, n}\right) \geq \sqrt{k-1}+\sqrt{l-1} .
$$

Assume now that we have a finite, connected, biregular, bipartite graph $X$ with valencies $q_{1}+1$ and $q_{2}+1$, and assume that $q_{1} \geq q_{2}$. Let $n_{i}$ denote the number of vertices of valency $q_{i}+1, i=1,2$, and set $V(X)=V_{1}(X) \sqcup V_{2}(X)$, where $V_{i}(X)$ consists of the vertices of $X$ with valency $q_{i}+1$. Then necessarily we have $n_{2} \geq n_{1}$. The adjacency matrix of the graph $X$ has the eigenvalues

$$
\begin{array}{r}
\operatorname{Spec}(X)=\{ \pm \lambda_{1}, \pm \lambda_{2}, \ldots \pm \lambda_{n_{1}}, \underbrace{0, \ldots, 0}_{n_{2}-n_{1}}\}, \\
\lambda_{1}=\sqrt{\left(1+q_{1}\right)\left(1+q_{2}\right)}>\lambda_{2} \geq \cdots \geq \lambda_{n_{1}} \geq 0 .
\end{array}
$$

Using the bound in Proposition 2.3, Hashimoto [10] (also Solé [22]) defines Ramanujan bigraphs as follows.

Definition 2.4. A finite, connected, biregular, bipartite graph with valencies $q_{1}+1$ and $q_{2}+1$ is called a Ramanujan bigraph if

$$
\left|\lambda^{2}-q_{1}-q_{2}\right| \leq 2 \sqrt{q_{1} q_{2}}
$$

for every $\lambda \in\left\{ \pm \lambda_{2}, \pm \lambda_{3}, \ldots, \pm \lambda_{n_{1}}\right\}$, where the notation is as in (2.4). In particular, this means that $\lambda_{n_{1}}>0$.

We call $X$ a weak Ramanujan bigraph if $\lambda_{n_{1}}>0$, in other words if $\operatorname{Ad}(X)$ has the eigenvalue 0 with multiplicity exactly $n_{2}-n_{1}$.

Next, we give an equivalent characterization for Ramanujan bigraphs. 
2.3. A cycle in a graph $X$ is an equivalence class of a closed path, where the equivalence is given by shifting the origin. Multiplication of paths is defined by concatenation. A cycle is primitive if it is not a power of a shorter cycle. If $X$ is a graph with fundamental group $\Gamma$ and $\rho: \Gamma \rightarrow U(n)$ is an $n$-dimensional unitary representation of $\Gamma$, we define, following [11, $\S 7$, the Zeta function of the graph $X$ attached to $\rho$.

Definition 2.5. The Zeta function of the graph $X$ attached to a representation $\rho$ of its fundamental group is given by

$$
Z_{X}(u ; \rho)=\prod_{\substack{P \text { primitive } \\ \text { cycle in } X}} \operatorname{det}\left(I_{n}-\rho(\langle P\rangle) u^{|P| / 2}\right)^{-1},
$$

where $|P|$ is the length of the geodesic cycle $P$ in $X$ and $\langle P\rangle$ is the corresponding conjugacy class in $\Gamma$. We denote the Zeta function of $X$ attached to the trivial representation of $\Gamma$ by $Z_{X}(u)$.

There is an explicit description in [10] of $Z_{X}(u)$ for a $\left(q_{1}+1, q_{2}+1\right)$-biregular graph $X$.

Theorem 2.6 ([10, Main Theorem (III)]). We have

$$
Z_{X}(u)^{-1}=(1-u)^{r-1}\left(1+q_{2} u\right)^{n_{2}-n_{1}} \prod_{j=1}^{n_{1}}\left(1-\left(\lambda_{j}^{2}-q_{1}-q_{2}\right) u+q_{1} q_{2} u^{2}\right),
$$

where $r=\operatorname{rank}$ of $\Gamma=|E(X)|-|V(X)|+1$.

The trivial zeros of $Z_{X}(u)^{-1}$ are $1,\left(q_{1} q_{2}\right)^{-1},-q_{2}{ }^{-1}$.

Definition 2.7. The Zeta function of a finite, connected, biregular, bipartite graph $X$ with valencies $q_{1}+1$ and $q_{2}+1$ is said to satisfy the Riemann Hypothesis if the nontrivial zeros of $Z_{X}(u)^{-1}$ satisfy the following property:

$$
\text { if } \Re(s) \in(0,1) \text { and } Z_{X}\left(\left(q_{1} q_{2}\right)^{-s}\right)^{-1}=0 \text {, then } \Re(s)=1 / 2 .
$$

The following result shows that the notion of a Ramanujan bigraph and the Riemann Hypothesis are equivalent. The proof is elementary.

Lemma 2.8. A finite, connected, biregular, bipartite graph is Ramanujan if and only if its Zeta function satisfies the Riemann Hypothesis.

Proof. Let $X$ be a $\left(q_{1}+1, q_{2}+1\right)$-biregular bipartite graph. If $X$ is Ramanujan, then $\left(\lambda^{2}-q_{1}-q_{2}\right)^{2}-4 q_{1} q_{2} \leq 0$, for $\lambda \in \operatorname{Spec}(X)$ with $\lambda^{2} \neq\left(1+q_{1}\right)\left(1+q_{2}\right)$. Condition (2.5) implies that each nonlinear, nontrivial term of $Z_{X}^{-1}(u)$ has complex conjugate roots of modulus $\left(q_{1} q_{2}\right)^{-1 / 2}$. Thus, since $\left|\left(q_{1} q_{2}\right)^{-s}\right|=\left(q_{1} q_{2}\right)^{-\Re(s)}, X$ Ramanujan implies that the complex solutions of $Z_{X}(u)$ are of the form $\left(q_{1} q_{2}\right)^{-s}$ with $\Re(s)=\frac{1}{2}$. The real solutions of $Z_{X}(u)$ are $1,-q_{2}^{-1},\left(q_{1} q_{2}\right)^{-1}$ (the trivial solutions), and $\pm\left(q_{1} q_{2}\right)^{-1 / 2}$ (if any eigenvalue satisfies the equality in (2.5)). Thus, $Z_{X}$ satisfies (2.8).

Conversely, assume that $Z_{X}$ satisfies (2.8), but suppose that $X$ is not Ramanujan. Then, there exists $\lambda \in \operatorname{Spec}(X)$ such that $\lambda^{2}<\left(q_{1}+1\right)\left(q_{2}+1\right)$ and $\left|\lambda^{2}-q_{1}-q_{2}\right|>$ $2 \sqrt{q_{1} q_{2}}$. This implies that the term $1-\left(\lambda^{2}-q_{1}-q_{2}\right) u+q_{1} q_{2} u^{2}$ in $Z_{X}(u)^{-1}$ has two real (nonequal) roots $u_{1,2}$. There are two cases. 
The first case is $\lambda^{2}-q_{1}-q_{2}>2 \sqrt{q_{1} q_{2}}$. Then $u_{1,2}$ are positive. We write the roots in the form $\left(q_{1} q_{2}\right)^{-s}$, where $s$ is real. Then we obtain $\lambda^{2}-q_{1}-q_{2}=\left(q_{1} q_{2}\right)^{1-s}+$ $\left(q_{1} q_{2}\right)^{s}$. The right hand side, as a function of $s$, has a minimum of $2 \sqrt{q_{1} q_{2}}$ at $s=1 / 2$. Thus, if $s \geq 1$, respectively $s \leq 0$, the right hand side is increasing, respectively decreasing, and we have $\lambda^{2}-q_{1}-q_{2}=\left(q_{1} q_{2}\right)^{1-s}+\left(q_{1} q_{2}\right)^{s} \geq 1+q_{1} q_{2}$. This contradicts our assumption that $\lambda^{2}<\left(q_{1}+1\right)\left(q_{2}+1\right)$. Thus, $s \in(0,1)$. Since the roots are positive, (2.8) implies that $s=\Re(s)=1 / 2$ and we have $\left(\lambda^{2}-q_{1}-q_{2}\right)^{2}=4 q_{1} q_{2}$. This however contradicts our assumption that $\left|\lambda^{2}-q_{1}-q_{2}\right|>2 \sqrt{q_{1} q_{2}}$.

The second case, $\lambda^{2}-q_{1}-q_{2}<-2 \sqrt{q_{1} q_{2}}$, is analogous.

\section{The Iwahori-Hecke Algebra of $S U(3)$}

3.1. Let $K$ be a local field with discrete valuation $\omega$. Let $q$ be the cardinality of the residue field. Let $L / K$ be an unramified separable quadratic extension. Let $\varpi$ be the uniformizer of $K$ and $\varpi_{1}$ the uniformizer of $L$. Let $\Xi=\omega\left(K^{\times}\right)(\subset \mathbb{R})$ be the value group of $K$. Since $L / K$ is unramified, $\Xi$ is also the value group of $L$. Consider the Hermitian form

$$
\Phi=\left(\begin{array}{ccc}
0 & 0 & 1 \\
0 & -1 & 0 \\
1 & 0 & 0
\end{array}\right)
$$

Set

$$
G=S U(3)=\left\{g \in S L_{3}(L) \mid g \Phi \bar{g}^{T}=\Phi\right\} .
$$

Let $S$ be a maximal torus in $G$ defined by

$$
S=\left\{\left(\begin{array}{ccc}
d & 0 & 0 \\
0 & 1 & 0 \\
0 & 0 & d^{-1}
\end{array}\right) \mid d \in K^{\times}\right\} .
$$

The root datum of $(G, S)$ is $\left(X, X^{\vee}, \Delta, \Delta^{\vee}\right)$. Here $X=X^{*}(S)=\operatorname{Hom}\left(S, K^{\times}\right)$, $X^{\vee}=X_{*}(S)=\operatorname{Hom}\left(K^{\times}, S\right)$ and $\Delta=\left\{a_{1}, a_{-1}, 2 a_{1}, 2 a_{-1}\right\}$, where $n a_{ \pm 1}: S \rightarrow K^{\times}$ is defined by

$$
n a_{ \pm 1}\left(\left(\begin{array}{ccc}
t & 0 & 0 \\
0 & 1 & 0 \\
0 & 0 & t^{-1}
\end{array}\right)\right)=t^{ \pm n}(n=1,2)
$$

The affine roots are

$$
\phi_{\mathrm{af}}=\left\{2 a_{i}+\gamma \mid i= \pm 1, \gamma \in \Xi\right\} \cup\left\{a_{i}+\frac{1}{2} \gamma \mid i= \pm 1, \gamma \in 2 \Xi\right\} .
$$

The inequalities $0<a_{1}<\omega\left(\varpi_{1}\right)$ define a chamber, and the corresponding basis is $\left\{a_{1}, 2 a_{-1}+\omega\left(\varpi_{1}\right)\right\}$. Let $\left\{s_{1}, s_{2}\right\}$ denote the corresponding reflections. They generate the affine Weyl group $W_{\text {af }}$, the infinite dihedral group.

Let $v_{1}$ be the vertex in the Dynkin diagram corresponding to the simple root $a_{1}$ and let $v_{2}$ be the vertex in the Dynkin diagram corresponding to the simple root $2 a_{-1}+\omega\left(\varpi_{1}\right)$. Following [6], the parameters of the root system are the integers $d\left(v_{1}\right)=3$ and $d\left(v_{2}\right)=1$. Set $q_{i}=q^{d\left(v_{i}\right)}, i=1,2$.

\footnotetext{
${ }^{1}$ If $L / K$ is ramified, the building of $S U(3)$ is a $(q+1)$-regular tree, and this case falls into the framework of 15 .
} 
3.2. Recall that a $G$-representation is called unitary if it is defined on a Hilbert space such that the inner product is $G$-invariant. Assume that $G$ acts on a space $Y$ which has a $G$-invariant measure. Then we can consider the Hilbert space $L^{2}(Y)$ of square integrable functions. This is a unitary representation of $G$ via the left regular action

$$
L_{g}(f)(y):=f\left(g^{-1} y\right), \quad g \in G, y \in Y, f \in L^{2}(Y) .
$$

When $Y$ is compact, the representation $L^{2}(Y)$ decomposes into a direct sum of irreducible unitary representations of $G$. The spaces that will occur naturally in our setting are of the form $Y=G / \Gamma$, where $\Gamma$ is a discrete co-compact subgroup of $G$.

Recall also that an irreducible unitary representation of $G$ is called a discrete series if it is a subrepresentation of $L^{2}(G)$, and it is called tempered if it occurs in the Hilbert direct integral decomposition of $L^{2}(G)$.

3.3. Let $\mathcal{H}(G)$ be the Hecke algebra of complex-valued, locally constant, compactly supported functions $f$ on $G$ with the product given by the convolution

$$
\left(f_{1} * f_{2}\right)(g):=\int_{G} f_{1}(x) f_{2}\left(x^{-1} g\right) d x, \quad f_{1}, f_{2} \in \mathcal{H}(G) .
$$

Here $d x$ is a Haar measure on $G$. Let $I$ denote an Iwahori subgroup of $G$. We normalize $d x$ such that the volume of $I$ is one.

If $(\pi, V)$ is a smooth representation of $G$, we obtain a representation of $\mathcal{H}(G)$ by defining

$$
\pi(f) v:=\int_{G} f(x) \pi(x) v d x, \quad f \in \mathcal{H}(G), v \in V .
$$

Define $\mathcal{H}(G, I)$ to be the subalgebra of $\mathcal{H}(G)$ consisting of functions bi-invariant under $I$. In the case $G=S U(3)$ (more generally, if $G$ is of simply connected type), the Bruhat-Tits decomposition is $G=\bigsqcup_{w \in W_{\mathrm{af}}} I w I$. Let $T_{1}$ and $T_{2}$ denote the characteristic functions of the double $I$-cosets with representatives $s_{1}$ and $s_{2}$, respectively. The algebra $\mathcal{H}(G, I)$ has the following Iwahori presentation: it is generated by $T_{1}$ and $T_{2}$ subject to the relations

$$
T_{i}^{2}=\left(q_{i}-1\right) T_{i}+q_{i}, \quad i=1,2 .
$$

Denote $V^{I}:=\{v \in V \mid \pi(i) v=v$, for all $i \in I\}$. Then $V^{I}$ is stable under the action of $\mathcal{H}(G, I)$ defined in (3.6), and we have the natural map (obtained by restriction) $\rho: \operatorname{Hom}_{G}(V, W) \longrightarrow \operatorname{Hom}_{\mathcal{H}(G, I)}\left(V^{I}, W^{I}\right)$, where $(\pi, V)$ and $\left(\pi^{\prime}, W\right)$ are smooth representations of $G$.

Theorem 3.1 (4). The functor $V \rightarrow V^{I}$ establishes an equivalence of categories between the category of smooth admissible $G$-representations which are generated by their I-fixed vectors and the category of finite-dimensional $\mathcal{H}(G, I)$-modules.

The inverse functor is described as follows. Let $(\varphi, E)$ be a representation of $\mathcal{H}(G, I)$. Set

$$
I(E)=C_{c}^{\infty}(G / I) \otimes_{\mathcal{H}(G, I)} E,
$$

where $C_{c}^{\infty}(G / I)$ is the space of compactly supported smooth functions which are right invariant under $I$. The tensor product makes sense since $\mathcal{H}(G, I)$ acts on $C_{c}^{\infty}(G / I)$ by convolution on the right and acts on the left on $E$ by the representation $\varphi$. We view $I(E)$ as a $G$-module, where $G$ acts on $C_{c}^{\infty}(G / I)$ by left translations. 
3.4. We describe explicitly the irreducible modules of $\mathcal{H}=\mathcal{H}(G, I)$. It will be convenient to use the Bernstein-Lusztig presentation of $\mathcal{H}$ ([17]) instead of the Iwahori presentation (3.7). As a $\mathbb{C}$-vector space, $\mathcal{H}=\mathcal{H}_{W} \otimes_{\mathbb{C}} \mathcal{A}$, where $\mathcal{H}_{W}=$ $\mathbb{C}[T] /\left\langle T^{2}=\left(q^{\lambda}-1\right) T+q^{\lambda}\right\rangle, \mathcal{A}=\mathbb{C}[\theta]$, and we have the commutation relation:

$$
\theta T-T \theta^{-1}=\left(q^{\lambda}-1\right) \theta+\left(q^{\frac{1}{2}\left(\lambda+\lambda^{*}\right)}-q^{\frac{1}{2}\left(\lambda-\lambda^{*}\right)}\right) .
$$

Here $\lambda$ and $\lambda^{*}$ are, in general, certain parameters, and in the case of $G=S U(3)$, $\lambda=3, \lambda^{*}=1$.

To go from the presentation (3.7) to (3.9), we will set

$$
T=T_{1}, \quad \theta=\frac{1}{\sqrt{q_{1} q_{2}}} T_{1} T_{2}, \quad q^{\lambda}=q_{1} \text { and } q^{\lambda^{*}}=q_{2} .
$$

For the remainder of the section we use the Bernstein-Lusztig presentation. The subalgebra $\mathcal{A}$ is abelian, and thus its irreducible representations are parameterized by the action of $\theta$. Assume that $\theta$ acts by a scalar $q^{\nu}, \nu \in \mathbb{C}$. Denote the corresponding character of $\mathcal{A}$ by $\mathbb{C}_{\nu}$. Define the principal series modules $X(\nu)$ as

$$
X(\nu)=\mathcal{H} \otimes_{\mathcal{A}} \mathbb{C}_{\nu}
$$

Since every irreducible $\mathcal{H}$-module has an $\mathcal{A}$-weight, it can be embedded into a principal series $X(\nu)$. A suitable basis for $X(\nu)$ is $\left\{(T+1) \otimes 1_{\nu},\left(T-q^{\lambda}\right) \otimes 1_{\nu}\right\}$. In this basis, the action of the two generators is

$$
\begin{aligned}
T & =\left(\begin{array}{cc}
q^{\lambda} & 0 \\
0 & -1
\end{array}\right) ; \\
\theta & =\frac{1}{q^{\lambda}+1} \\
& \times\left(\begin{array}{cc}
q^{\lambda-\nu}+q^{\lambda+\nu}+\left(q^{\frac{1}{2}\left(\lambda+\lambda^{*}\right)}-q^{\frac{1}{2}\left(\lambda-\lambda^{*}\right)}\right) & q^{\lambda-\nu}-q^{\nu}+\left(q^{\frac{1}{2}\left(\lambda+\lambda^{*}\right)}-q^{\frac{1}{2}\left(\lambda-\lambda^{*}\right)}\right) \\
q^{-\nu}-q^{\lambda+\nu}-\left(q^{\frac{1}{2}\left(\lambda+\lambda^{*}\right)}-q^{\frac{1}{2}\left(\lambda-\lambda^{*}\right)}\right) & q^{-\nu}+q^{\nu}-\left(q^{\frac{1}{2}\left(\lambda+\lambda^{*}\right)}-q^{\frac{1}{2}\left(\lambda-\lambda^{*}\right)}\right)
\end{array}\right) .
\end{aligned}
$$

To find this matrix for $\theta$, one multiplies the basis elements on the left by $\theta$ and then commutes past $T$ using equation (3.9). The eigenvalues of $\theta$ are $q^{\nu}$ and $q^{-\nu}$. These are the $\mathcal{A}$-weights of the module $X(\nu)$.

We summarize the classification of irreducible $\mathcal{H}$-modules.

Proposition 3.2. (1) Every irreducible $\mathcal{H}$-module appears as a subquotient of a two-dimensional principal series $X(\nu)$, where $\nu \in \mathbb{C} /(2 \pi i / \log q)$.

(2) The module $X(\nu)$ is irreducible unless $\nu \in\left\{ \pm \frac{\lambda+\lambda^{*}}{2}, \pm \frac{\lambda-\lambda^{*}}{2}+\frac{\pi i}{\log q}\right\}$.

(3) The one-dimensional $\mathcal{H}$-modules are

$$
\begin{array}{ll}
\text { St }=\left(T=-1, \theta=q^{-\frac{1}{2}\left(\lambda+\lambda^{*}\right)}\right) ; & \mathrm{ds}=\left(T=-1, \theta=-q^{\frac{1}{2}\left(\lambda^{*}-\lambda\right)}\right) ; \\
\text { sph }=\left(T=q^{\lambda}, \theta=q^{\frac{1}{2}\left(\lambda+\lambda^{*}\right)}\right) ; \quad \text { nt }=\left(T=q^{\lambda}, \theta=-q^{\frac{1}{2}\left(\lambda-\lambda^{*}\right)}\right) .
\end{array}
$$

Proof. To find the one-dimensional modules, and implicitly determine when $X(\nu)$ is reducible, notice that if $T$ acts by a scalar, there are two possibilities, either $T=-1$ or $T=q^{\lambda}$. Solving in the commutation relation (3.9) for $\theta$, we obtain these four one-dimensional representations. 
The center of $\mathcal{H}$ can be shown to equal $Z(\mathcal{H})=\mathbb{C}\left[\theta+\theta^{-1}\right]$. (This is an easy particular case of a more general result of Bernstein and Lusztig [17, Proposition 3.11].) Therefore, if $(\pi, V)$ is a subquotient of $X(\nu)$, the central character of $\pi$ is determined by $\nu$.

3.5. Later we will need to know the unitary irreducible modules of $\mathcal{H}$. The algebra $\mathcal{H}=\mathcal{H}(G, I)$ has a natural $*$-operation defined on functions by

$$
f^{*}(g):=\overline{f\left(g^{-1}\right)}, \quad f \in \mathcal{H}(G, I) .
$$

Calculated on the generators $T, \theta$, this becomes

$$
T^{*}=T^{-1}, \quad \theta^{*}=T \theta T^{-1} .
$$

Definition 3.3. (1) We say that an $\mathcal{H}$-module $(\pi, V)$ is $(*-)$ unitary if it admits a Hermitian form $\langle$,$\rangle such that$

$$
\langle\pi(x) v, w\rangle=\left\langle v, \pi\left(x^{*}\right) w\right\rangle, \quad \text { for all } x \in \mathcal{H}, v, w \in V .
$$

(2) (Casselman's criterion) An $\mathcal{H}$-module $(\pi, V)$ is called tempered (resp. a discrete series) if $|\chi| \leq 1$ (resp. $|\chi|<1$ ), for every $\mathcal{A}$-weight $\chi$ of $\pi$.

Proposition 3.4. $\quad$ (1) In the correspondence $V \rightarrow V^{I}$ of Theorem 3.1, the $G$ representation $V$ is tempered (resp. discrete series, unitary) if and only if the $\mathcal{H}(G, I)$-representation $V^{I}$ is tempered (resp. a discrete series, unitary).

(2) The discrete series $\mathcal{H}$-modules are the one-dimensional modules $\mathrm{St}$ and $\mathrm{ds}$. The tempered $\mathcal{H}$-modules, that are not discrete series, are the direct summands of $X(\nu)$, for $\Re \nu=0$.

(3) The unitary $\mathcal{H}$-modules are the subquotients of the principal series $X(\nu)$ in one of the following cases:

- $\nu \in \mathbb{R}$ and $|\nu| \leq \frac{\lambda+\lambda^{*}}{2}$;

- $\Re \nu=0$;

$$
\text { - } \Im \nu=\frac{\pi i}{\log q} \text { and }|\Re \nu| \leq \frac{\lambda-\lambda^{*}}{2} \text {. }
$$

Proof. (1) The claim about tempered modules and discrete series is well known (see [7). The correspondence for unitary modules is a particular case of [3, Theorem 1], which generalizes results of Barbasch and Moy.

(2) This is obvious from Proposition 3.2. since in our notation $\lambda>\lambda^{*}$.

(3) One way to prove this is to make use of [3], where we can reduce this question to one about Lusztig's graded Hecke algebra [17. (It is of course possible to prove the claim directly, without appealing to the graded Hecke algebra.)

Let $\mathbb{H}_{\mu}$ be the $\mathbb{C}$-algebra with unit generated by $s$ and $\epsilon$ subject to the relations

$$
s^{2}=1, \quad \epsilon \cdot s+s \cdot \epsilon=2 \mu,
$$

where $\mu$ is a nonnegative scalar. This algebra has a $*$-operation as well, defined on the generators by

$$
s^{*}=s, \quad \epsilon^{*}=\epsilon+\mu s,
$$

and again we can talk about unitary modules. The classification of the irreducible $\mathbb{H}_{\mu}$-modules is similar, but simpler, than the one for $\mathcal{H}$-modules. More precisely, denote by $\mathbb{C}[W]=\mathbb{C}[s]$ and $\mathbb{A}=\mathbb{C}[\epsilon]$, and define the principal series $\bar{X}(\nu)=$ $\mathbb{H}_{\mu} \otimes_{\mathbb{A}} \mathbb{C}_{\nu}$, where $\mathbb{C}_{\nu}$ is a character of $\mathbb{A}, \nu \in \mathbb{C}$. Then, we have:

- every irreducible $\mathbb{H}_{\mu}$-module is a subquotient of a $\bar{X}(\nu)$;

- $\bar{X}(\nu)$ is irreducible unless $\nu= \pm \mu$; 
- the one-dimensional irreducible $\mathbb{H}_{\mu}$-modules are $(s=1, \epsilon=\mu)$ and $(s=$ $-1, \epsilon=-\mu)$.

We say that an $\mathbb{H}_{\mu}$-module has real central character if the eigenvalues of $\epsilon$ are real scalars or, equivalently, if the parameter $\nu$ is in $\mathbb{R}$.

The unitary modules are easily determined too. A Hermitian form on $\bar{X}(\nu)$ is equivalent with an intertwining operator between $\bar{X}(\nu)$ and the Hermitian dual of $\bar{X}(\nu)$, which is $\bar{X}(-\bar{\nu})$. When $\nu \geq 0$, the operator is

$$
A(\nu): \bar{X}(\nu) \rightarrow \bar{X}(-\nu), \quad A(\nu)\left(x \otimes 1_{\nu}\right)=\frac{1}{\nu+\mu}(\epsilon \cdot s+\mu) \cdot x \otimes 1_{-\nu}
$$

Using the basis $\left\{(1+s) \otimes 1_{\nu},(1-s) \otimes 1_{\nu}\right\}$, one can immediately find that

$$
[A(\nu)]=\left(\begin{array}{cc}
1 & 0 \\
0 & \frac{\mu-\nu}{\mu+\nu}
\end{array}\right) .
$$

This means that $A(\nu), \nu \geq 0$, is positive semidefinite if and only if $0 \leq \nu \leq \mu$.

The relation with $\mathcal{H}$ is as follows. Fix $\xi \in i(\mathbb{R} / \log q)$. Then following Lusztig [17. §4.1], one defines an ideal $\mathcal{I}_{\xi}$ of $\mathcal{A}$ such that the associated graded object to the filtration $\mathcal{H} \supset \mathcal{H} \cdot \mathcal{I}_{\xi} \supset \mathcal{H} \cdot \mathcal{I}_{\xi}^{2} \supset \ldots$ is a graded Hecke algebra of the type defined here. The point is that one obtains a natural bijection between irreducible $\mathcal{H}$-modules parameterized by $\nu$ such that $\Im \nu=\xi$ and $\mathbb{H}_{\mu}$-modules parameterized by $\Re \nu$ ([17, Corollary 10.8]). This correspondence preserves unitarity too. The two interesting cases are $\xi=0$, in which case the corresponding graded algebra is $\mathbb{H}_{\mu}$, $\mu=\frac{\lambda+\lambda^{*}}{2}$, and the case $\xi=\frac{\pi i}{\log q}$ when the corresponding graded algebra is $\mathbb{H}_{\mu}$, $\mu=\frac{\lambda-\lambda^{*}}{2}$. This explains the unitary "complementary series" in our proposition. (When $\xi \neq 0, \frac{\pi i}{\log q}$, the corresponding graded Hecke algebra is not one of the $\mathbb{H}_{\mu}$ 's, but rather just an abelian algebra. Consequently, its unitary dual with "real central character" is a point, corresponding to the tempered module $X(\nu), \Re \nu=0$.)

\section{Adjacency operators and quotients of the $S U(3)$-Tree}

We retain the notation from the previous sections, particularly 3.1 . The BruhatTits tree of $S U(3)$ is a $\left(q^{3}+1, q+1\right)$-biregular graph, where $q$ is the cardinality of the residue field of $K$. We denote it by $\tilde{X}$. (One obtains the same graph if one considers $U(3)$ instead of $S U(3)$.)

Recall that $I$ is an Iwahori subgroup. Set $U_{i}:=I \cup I s_{i} I, i=1,2$. The subgroups $U_{i}, i=1,2$, contain $I$ properly and are representatives of the $G$-conjugacy classes of maximal (open) compact subgroups. In fact, $I=U_{1} \cap U_{2}$. Define a length function on $G$ as in 11 and let $U\left(=U_{1}\right)$ be the set of elements of length 0 . Let $\Gamma$ be a discrete co-compact subgroup of $G$ as in 11] such that $\Gamma$ is torsion free, $\Gamma \cap x^{-1} U x=\{1\}$ for any $x \in G$, and $\#(U \backslash G / \Gamma)<\infty$.

Let $X$ be the quotient graph $\tilde{X} / \Gamma$. Then $X$ is a finite, connected, biregular, bipartite graph with valencies $q^{3}+1$ and $q+1$, and therefore the discussion in the previous sections applies to $X$. We would like to investigate when $X$ is a Ramanujan bigraph. For this, we will make use of the Iwahori-Hecke algebra $\mathcal{H}=\mathcal{H}(G, I)$ defined before.

4.1. We summarize the results from [10, $\S 5,6]$ establishing the relationship between the algebra generated by the colored edge adjacency operators and $\mathcal{H}(G, I)$. 
Let $\mathbb{Z}[E(\tilde{X})]$ be the free $\mathbb{Z}$-module over the set $E(\tilde{X})$ of edges of $\tilde{X}$. We define $T_{1}, T_{2}$ to be the elements of $\operatorname{End}(\mathbb{Z}[E(\tilde{X})])$ given by

$$
T_{i}(e):=\sum_{e^{\prime} \in \tilde{E}_{i}(e) \backslash\{e\}} e^{\prime} \quad(i=1,2)
$$

where $\tilde{E}_{i}(e)$ is the set of edges in $\tilde{X}$ incident to the vertex of $e$ that lies in $V_{i}$.

Let $\mathbb{C}[E(X)]$ be the space of $\mathbb{C}$-valued functions on $E(X)$, the edges of $X$. It carries an inner product

$$
\left(f, f^{\prime}\right):=\sum_{e \in E(X)}\left(f(e), f^{\prime}(e)\right)_{\mathbb{C}}
$$

where $(,)_{\mathbb{C}}$ is the usual inner product in $\mathbb{C}$. Since the action of $\Gamma$ preserves the incidences in $\tilde{X}$, the operators $T_{1}, T_{2}$ induce naturally endomorphisms on $\mathbb{C}[E(X)]$ (denoted by $T_{1}, T_{2}$ again),

$$
\left(T_{i} f\right)(e):=\sum_{e^{\prime} \in E_{i}(e)} f\left(e^{\prime}\right)-f(e) \quad(i=1,2) .
$$

Moreover, $T_{1}, T_{2}$ are isometries with respect to the inner product (4.2). Recall the Zeta function $Z_{X}$ from definition [2.5. By [10, Main Theorem (I)], we have

$$
Z_{X}(u)^{-1}=\operatorname{det}\left(I-T_{1} T_{2} u\right) .
$$

Let $\mathbb{C}\left[T_{1}, T_{2}\right]$ be the $\mathbb{C}$-subalgebra of $\operatorname{End}_{\mathbb{C}}(\mathbb{Z}[E(X)])$ generated by $T_{1}, T_{2}$. It is a noncommutative ring of polynomials in $T_{1}, T_{2}$ with fundamental relations

$$
T_{i}^{2}=\left(q_{i}-1\right) T_{i}+q_{i} \quad(i=1,2) .
$$

This means that $\mathbb{C}\left[T_{1}, T_{2}\right] \cong \mathcal{H}(G, I)$. In the previous section (Proposition 3.2), we saw that the irreducible $\mathcal{H}(G, I)$-modules lie in two-dimensional principal series $X(\nu)$, where $\nu$ is in the parameter space $\mathbb{C} /(2 \pi i / \log q)$. To every irreducible Hecke module $\varphi$, one associates a characteristic polynomial,

$$
\begin{aligned}
p_{\varphi}(u) & =\operatorname{det}\left(1-\varphi\left(T_{1} T_{2}\right) u\right)=\operatorname{det}\left(1-\sqrt{q_{1} q_{2}} \varphi(\theta) u\right) \\
& =1-\sqrt{q_{1} q_{2}} \operatorname{Tr}_{\varphi}(\theta) u+q_{1} q_{2} u^{2},
\end{aligned}
$$

when $\varphi$ is two-dimensional; here $\theta$ is the generator from (3.10). Notice that the one-dimensional modules have the following characteristic polynomials:

$$
\begin{array}{ll}
p_{\text {St }}(u)=1-u ; & p_{\text {ds }}(u)=1+q_{2} u ; \\
p_{\text {sph }}(u)=1-q_{1} q_{2} u ; & p_{\text {nt }}(u)=1+q_{1} u .
\end{array}
$$

We need to determine which representations $\varphi$ of $\mathcal{H}$ have the property that $p_{\varphi}(u)$ occurs in $Z_{X}(u)^{-1}$. From (2.7), one immediately sees that $p_{\mathrm{St}}(u)$ occurs with multiplicity exactly $r, p_{\text {sph }}(u)$ has multiplicity one, while $p_{\mathrm{ds}}(u)$ occurs with multiplicity at least $n_{2}-n_{1}$. More precisely, $p_{\mathrm{ds}}(u)$ occurs in $Z_{X}(u)^{-1}$ with multiplicity exactly $n_{2}-n_{1}$ if and only if $X$ is weak Ramanujan. The only way the multiplicity of $p_{\mathrm{ds}}(u)$ is greater than $n_{2}-n_{1}$ is if $p_{\text {nt }}(u)$ occurs in $Z_{X}(u)^{-1}$. 
4.2. Recall the space $L^{2}(G / \Gamma)$ with the left regular representation of $G$, where $\Gamma$ is subject to the same assumptions as in the beginning of 4 . Consider the subspace of $I$-invariant vectors $L^{2}(G / \Gamma)^{I}=L^{2}(I \backslash G / \Gamma)=\mathbb{C}[E(X)]$. If $\pi$ is a unitary irreducible representation of $G$, let $m_{\Gamma}(\pi)$ represent the multiplicity of $\pi$ in $L^{2}(G / \Gamma)^{I}$. The two discrete series of $G$, whose $I$-fixed vectors form the $\mathcal{H}(G, I)$-modules $S t$ and ds, occur in this space. Since $G / \Gamma$ is compact, the trivial representation also appears.

Let $(\pi, V)$ be an irreducible unitary smooth representation of $G$ such that $V^{I} \neq$ $\{0\}$. Let $\left(\varphi, V^{I}\right)$ be the corresponding irreducible representation of $\mathcal{H}(G, I)$. By Proposition 3.4, this is a unitary finite-dimensional module of $\mathcal{H}(G, I)$.

Putting these together, one finds [10, Main Theorem (IV)]):

$$
m_{\Gamma}(\pi)=\text { multiplicity of } p_{\varphi}(u) \text { in } Z_{X}(u)^{-1} \text {. }
$$

If $m_{\Gamma}(\pi)>0$, and $\varphi \notin\{\mathrm{St}, \mathrm{ds}\}$, we see that the corresponding factor in $Z_{X}(u)^{-1}$ is $p_{\varphi}(u)=1-\sqrt{q_{1} q_{2}} \operatorname{Tr}_{\varphi}(\theta) u+q_{1} q_{2} u^{2}$ on the one hand, but on the other hand is of the form $\left(1-\left(\lambda_{j}^{2}-q_{1}-q_{2}\right) u+q_{1} q_{2} u^{2}\right)$. This means that $\operatorname{Tr}_{\varphi}(\theta)=\frac{\lambda_{j}^{2}-q_{1}-q_{2}}{\sqrt{q_{1} q_{2}}}$, for some eigenvalue $\lambda_{j}$ of $\operatorname{Ad}(X)$. The Ramanujan condition (2.5) is therefore equivalent to:

$$
\left|\operatorname{Tr}_{\varphi}(\theta)\right| \leq 2 \text {, i.e., } \varphi \text { is tempered (by Proposition } 3.4 \text { ). }
$$

We phrase this condition as follows.

Conjecture 4.1 (Ramanujan conjecture2). Every nontrivial irreducible unitary $\mathcal{H}(G, I)$-module that appears in the decomposition of $L^{2}(G / \Gamma)^{I}=L^{2}(I \backslash G / \Gamma)$ is tempered.

Recall that the explicit description of unitary $\mathcal{H}(G, I)$-modules is given in Proposition 3.4. We have obtained the following criterion:

Theorem 4.2. Let $G=S U(3)$ be the unitary group in three variables defined in \$3.1. Let $\Gamma$ be a discrete, co-compact subgroup of $G$ which acts on $G$ without fixed points. If $\tilde{X}$ is the Bruhat-Tits tree associated with $G$, then the building quotient $X=\tilde{X} / \Gamma$ is a Ramanujan bigraph if and only if $\Gamma$ satisfies Conjecture 4.1.

4.3. In this section, let $k$ denote a global field of characteristic 0 , and let $E / k$ be a separable quadratic extension. Denote by ${ }^{-}$the conjugation of $E$ with respect to $k$. Let $D$ be a simple 9-dimensional algebra with center $E$ and norm $N$, and let $\alpha: D \rightarrow D$ be an anti-automorphism such that $\alpha(x)=\bar{x}$, for all $x \in E$ (i.e., an automorphism of the second kind). For the classification of pairs $(D, \alpha)$ we refer to [14, Theorems (3.1) and (19.6)]. Every such $D$ is a cyclic algebra over $E$ [14, Theorem (19.2) and Proposition (19.15)]. More precisely, every $(D, \alpha)$ arises as follows. Let $L$ be a 3 -dimensional algebra over $E$, which is $\mathbb{Z} / 3 \mathbb{Z}$-Galois over $E$ and $S_{3}$-Galois over $k$. This means that $L$ has an automorphism $\sigma$ of order 3 and an automorphism $\iota$ of order 2 , such that $\langle\sigma, \iota\rangle \cong S_{3},\left.\iota\right|_{E}={ }^{-}$, and the fixed points of $\sigma$ in $L$ are $L^{\sigma}=E$, while the fixed points of $\langle\sigma, \iota\rangle$ in $L$ are $L^{\langle\sigma, \iota\rangle}=k$. Notice that $\sigma \circ \iota=\iota \circ \sigma^{2}$ on $L$. Set

$$
\begin{aligned}
& D=L \oplus L z \oplus L z^{2}, \text { with } \\
& z \ell=\sigma(\ell) z, \quad z^{3}=a,
\end{aligned}
$$

\footnotetext{
${ }^{2}$ The term "conjecture" could be misleading since the existence of (local) Arthur representations 1] implies that this condition may fail for certain groups $\Gamma$ (even if $\Gamma$ is assumed to be a congruence subgroup). Perhaps a better name would be the "Ramanujan condition" (or "Ramanujan hypothesis"), but we decided to follow the classical terminology from $P G L_{2}\left(\mathbb{Q}_{p}\right)$.
} 
for some fixed $a \in k^{\times}$, and set the involution $\alpha$ to be

$$
\alpha(z)=z, \quad \alpha(\ell)=\iota(\ell), \text { for all } \ell \in L
$$

Define $\mathbb{G}$ to be an inner form of $S U(3)$ determined by $(D, \alpha)$, more precisely $\mathbb{G}=\left\{g \in D^{*}: \alpha(g) g=1, N(g)=1\right\}$. For example, if $D \cong M_{3}(E)$, meaning in (4.9) that $L \cong E^{3}$, then $\alpha(g)=\Phi \bar{g}^{T} \Phi^{-1}$, for some Hermitian form $\Phi$. The quasisplit form from section 3.1 is such an example. For the explicit connection with the realizations (4.9, 4.10), we refer to [14, Example (19.17)].

For every place $v$, we denote $\mathbb{G}_{v}=\mathbb{G}\left(k_{v}\right)$ and $D_{v}=D \otimes_{k} k_{v}$. For the finite places $v$ of $k$, we have the following possible cases (see [20] or [19, §1.9]) for the group $\mathbb{G}_{v}=\left\{g \in D_{v}^{*}: \alpha(g) g=1, N(g)=1\right\}$ over the local field $k_{v}$ :

(a) if $v$ splits into $v=w \bar{w}$ in $E$, then $D_{v}=D_{w} \oplus D_{\bar{w}}$, and $\mathbb{G}_{v} \cong D_{w}^{1} \cong D_{\bar{w}}^{1}$ (norm one units);

(b) if $v$ remains prime in $E$, then $\mathbb{G}_{v} \cong G$, where $G$ is the quasisplit unitary group over $k_{v}$ defined in section 3.1

In order to use the results of [19, we restate the previous criterion, Theorem 4.2 . from a global perspective.

Theorem 4.3. Let $v$ be a finite place of $k$ with $E_{v} / k_{v}$ unramified, and such that at the place $v, \mathbb{G}_{v} \cong G$. Let $\Gamma_{v}$ be a discrete, co-compact subgroup of $\mathbb{G}_{v}$ which acts on $\mathbb{G}_{v}$ without fixed points. If $\tilde{X}_{v}$ is the Bruhat-Tits tree associated with $\mathbb{G}_{v}$, then the building quotient $X_{v}=\tilde{X}_{v} / \Gamma_{v}$ is a Ramanujan bigraph if and only if $\mathbb{G}_{v}$ satisfies Conjecture 4.1.

By the Strong Approximation Theorem (13), verifying Conjecture 4.1 is equivalent with deciding which representations of $\mathbb{G}_{v}=G$ with $I$-fixed vectors occur as local components of the automorphic representations of $\mathbb{G}$ over the adeles. We explain this connection now.

In order to simplify notation, let us assume that $k=\mathbb{Q}$, and we will denote a prime, as usual, by $p$. Assume that $E / \mathbb{Q}$ is imaginary. Let $\mathbb{A}$ denote the ring of adeles of $\mathbb{Q}$, and let $\mathbb{A}_{p}$ be the ring of adeles without the factor at the place $p$. Let $\mathbb{Z}$ be the ring of integers of $\mathbb{Q}$.

Fix a finite prime $p$ as in Theorem 4.3. Since we are looking at forms of unitary groups of odd order, there exists a $\mathbb{Q}$-group $\mathbb{G}$ such that $\mathbb{G}_{p} \cong G$; in particular, this means that $\mathbb{G}_{p}$ is not compact. (In fact, $\mathbb{G}_{v} \cong G$ at almost all finite places.) Moreover, we may require that $\mathbb{G}(\mathbb{R})$ be compact. Such groups $\mathbb{G}$ exist; see for example [8, §3.3].

Let $\mathbb{Z}\left[p^{-1}\right]$ denote the subring of $\mathbb{Q}$ consisting of all rational numbers whose denominators are powers of $p$. One thinks of $\mathbb{Z}\left[p^{-1}\right]$ as being embedded diagonally into $\mathbb{R} \times \mathbb{Q}_{p}$. A theorem of Borel [5] implies that $\Gamma_{p}=\mathbb{G}\left(\mathbb{Z}\left[p^{-1}\right]\right)$ is a lattice in $\mathbb{G}(\mathbb{R}) \times \mathbb{G}_{p}$. For every positive integer $n$ coprime to $p$, define

$$
\Gamma(n)=\operatorname{ker}\left(\mathbb{G}\left(\mathbb{Z}\left[p^{-1}\right]\right) \rightarrow \mathbb{G}\left(\mathbb{Z}\left[p^{-1}\right] / n \mathbb{Z}\left[p^{-1}\right]\right)\right), \quad \Gamma_{p}(n)=\Gamma(n) \cap \mathbb{G}_{p} .
$$

Therefore $\Gamma_{p}(n)$ is a discrete co-compact lattice in $\mathbb{G}_{p}$. 
Assume that the factorization of $n$ in $\mathbb{Z}$ into primes is $n=\prod_{i=1}^{r} p_{i}^{d_{i}}$. Define

$$
\begin{aligned}
& K_{p_{i}}=\operatorname{ker}\left(\mathbb{G}\left(\mathbb{Z}_{p_{i}}\right) \rightarrow \mathbb{G}\left(\mathbb{Z}_{p_{i}} / p_{i}{ }^{{ }} \mathbb{Z}_{p_{i}}\right)\right), \quad i=1, \ldots, r ; \\
& K_{\ell}=\mathbb{G}\left(\mathbb{Z}_{\ell}\right), \quad \ell \neq p_{i}, p, \quad \ell<\infty ; \\
& K_{p}=I_{p} \quad \text { (the Iwahori subgroup); } \\
& \left.K_{\infty}=S U(3) \quad \text { (the compact unitary group over } \mathbb{R}\right) .
\end{aligned}
$$

Set

$$
K^{n}=\prod_{l \leq \infty} K_{\ell} \quad \text { and } \quad K_{(p)}^{n}=\prod_{l \leq \infty, \ell \neq p} K_{\ell} .
$$

These are compact open subgroups of $\mathbb{G}(\mathbb{A})$ and $\mathbb{G}\left(\mathbb{A}_{p}\right)$ respectively. Since we assumed that $\mathbb{G}(\mathbb{R})=K_{\infty}$, in other words that $\mathbb{G}(\mathbb{R})$ is compact, we have $\mathbb{G}(\mathbb{Q}) \cap$ $K_{(p)}^{n}=\Gamma_{p}(n)$.

By [5, Theorem 5.1], the number of double cosets in

$$
K^{n} \backslash \mathbb{G}(\mathbb{A}) / \mathbb{G}(\mathbb{Q})
$$

is finite and, therefore, the number of double cosets in

$$
K_{(p)}^{n} \mathbb{G}_{p} \backslash \mathbb{G}(\mathbb{A}) / \mathbb{G}(\mathbb{Q})
$$

is finite. Let $\left\{x_{1}, \ldots, x_{s}\right\}$ be a set of representatives of these cosets. We have

$$
\mathbb{G}(\mathbb{A})=\bigcup_{i=1}^{s}\left(K_{(p)}^{n} \mathbb{G}_{p}\right) x_{i} \mathbb{G}(\mathbb{Q})
$$

Consider the group $\Gamma_{i, n}^{\prime}=K_{(p)}^{n} \mathbb{G}_{p} \cap x_{i} \mathbb{G} x_{i}^{-1}$. Note that when $x_{i}$ is the identity, $\Gamma_{i, n}^{\prime}$ is precisely $\Gamma(n)$ defined in (4.11). Since $K_{\infty}$ is compact, the projection of $\Gamma_{i, n}^{\prime}$ on $\mathbb{G}_{p}$, denoted by $\Gamma_{i, p, n}^{\prime}$, remains a discrete subgroup. Each group $\Gamma_{i, p, n}^{\prime}$ is finitely generated. Then, by [21, Lemma 8], $\Gamma_{i, p, n}^{\prime}$ has a normal subgroup $\Gamma_{i, p, n}$ of finite index which has no nontrivial elements of finite order. Thus, each element of $\Gamma_{i, p, n}$ different from the identity acts on $K_{p} \backslash \mathbb{G}_{p}$ without fixed points. As noted before, when $x_{i}$ is the identity element, $\Gamma_{i, p, n}$ is precisely $\Gamma_{p}(n)$. We have

$$
L^{2}\left(K_{(p)}^{n} \backslash \mathbb{G}(\mathbb{A}) / \mathbb{G}(\mathbb{Q})\right)=\bigotimes_{i=1}^{s} L^{2}\left(\mathbb{G}_{p} / \Gamma_{i, p, n}\right)
$$

In other words, every irreducible $\mathbb{G}_{p}$-representation that occurs in $L^{2}\left(\mathbb{G}_{p} / \Gamma_{i, p, n}\right)$ must be the local factor of an automorphic representation of $\mathbb{G}$. Define

$$
X_{i, p, n}:=K_{p} \backslash \mathbb{G}_{p} / \Gamma_{i, p, n} .
$$

In [19, Rogawski completely classifies the automorphic representations of $\mathbb{G}$. (A convenient reference for the needed results from [19] is [2, §11].) Recall that we are assuming that at the place $p, \mathbb{G}_{p}=G$. In turns out that the only nontrivial nontempered automorphic representation of $\mathbb{G}_{p}$ is the unitary representation whose $I$-fixed vectors form the $\mathcal{H}(G, I)$-module nt (notation as in Proposition 3.2). By the discussion at the end of 44.1 , this result of [19] implies the following equivalence:

Corollary 4.4. Assume that $\mathbb{G}(\mathbb{R})$ is compact and that $\mathbb{G}_{p}=G$. Let $X=X_{i, p, n}$ be a finite bigraph constructed in (4.16). Then $X$ is Ramanujan if and only if $X$ is weak Ramanujan. 
Recall that "weak Ramanujan" is a simple condition on the multiplicity of 0 as an eigenvalue of $\operatorname{Ad}(X)$ (Definition 2.4).

Moreover, again from [19], we have:

Theorem 4.5 ([19, Theorem 14.6.3]). Assume that $D \neq M_{3}(E)$. Then no irreducible representation $(\pi, V)$ of $G=\mathbb{G}_{p}$ such that $V^{I} \cong \mathrm{nt}$ as $\mathcal{H}(G, I)$-modules occurs as the local component of an automorphic representation of $\mathbb{G}$.

Therefore comparing this with Theorem 4.3, we have the following corollary.

Corollary 4.6. Assume that $D \neq M_{3}(E)$ and that $\mathbb{G}(\mathbb{R})$ is compact. Then the family of finite tree quotients $X_{i, p, n}$ from (4.16) is an infinite family of Ramanujan bigraphs.

\section{ACKNOWLEDGMENTS}

The authors thank Jon Rogawski for suggesting the problem and Gordan Savin for helpful conversations about the material in 4.3

\section{REFERENCES}

1. James Arthur, Unipotent automorphic representations: conjectures, Astérisque 171-172 (1989), 13-71. MR.1021499 (91f:22030)

2. C. Ballantine, Ramanujan type buildings, Canad. J. Math. 52 (2000), no. 6, 1121-1148. MR 1794299 (2001j:11027)

3. D. Barbasch, D. Ciubotaru, Unitary functorial correspondences for p-adic groups, preprint, arXiv:0909.5241.

4. A. Borel, Admissible representations of a semi-simple group over a local field with vectors fixed under an Iwahori subgroup, Invent. Math. 35 (1976), 233-259. MR0444849 (56:3196)

5. A. Borel, Some finiteness properties of adele groups over number fields, Publ. Math. IHES 16 (1963), 5-30. MR0202718(34:2578)

6. P. Cartier, Representations of p-Adic Groups: A Survey, Proc. Symp. Pure Math., 33, Vol. 1, Amer. Math. Soc., 1979, 111-155. MR546593 (81e:22029)

7. W. Casselman, Introduction to admissible representations of $p$-adic groups, http://www. math . ubc.ca/ cass/research.html

8. L. Clozel, M. Harris, R. Taylor, Automorphy for some l-adic lifts of automorphic mod $l$ Galois representations, Publ. Math. Inst. Hautes Études Sci. 108 (2008), 1-181. MR2470687 (2010j:11082)

9. K. Feng, W.-C.W. Li, Spectra of hypergraphs and applications, J. Number Theory 60, no. 1 (1996), 1-22. MR1405722 (97f:05128)

10. K. Hashimoto, Zeta Functions of Finite Graphs and Representations of p-Adic Groups, Advanced Studies in Pure Mathematics, 15, Academic Press, Boston, MA, 1989, Automorphic Forms and Geometry of Arithmetic Varieties, 211-280. MR.1040609 (91i:11057)

11. K. Hashimoto and A. Hori, Selberg-Ihara's Zeta Function for p-adic Discrete Subgroups, Advanced Studies in Pure Mathematics 15, Academic Press, Boston, MA, 1989, Automorphic Forms and Geometry of Arithmetic Varieties, 171-210.

12. Y. Ihara, Discrete subgroups of $P L\left(2, k_{p}\right)$, Proc. Symp. Pure Math. IX, Amer. Math. Soc., 1966, 272-278. MR 0205952 (34:5777)

13. M. Kneser, Strong approximation, Proc. Symp. Pure Math. IX, Amer. Math. Soc., 1966, 187-196. MR0213361 (35:4225)

14. M.-A. Knus, A. Merkurjev, M. Rost, J.-P. Tignol, The book of involutions, Amer. Math. Soc. Coll. Publ., 44, Amer. Math. Soc., 1998. MR1632779 (2000a:16031)

15. A. Lubotzky, R. Phillips, P. Sarnak, Ramanujan graphs, Combinatorica 8 (1988), 261-277. MR:963118 (89m:05099)

16. A. Lubotzky, Discrete Groups, Expanding Graphs and Invariant Measures, Progress in Math., 125, Birkhäuser Verlag, 1994. MR1308046 (96g:22018)

17. G. Lusztig, Affine Hecke algebras and their graded version, J. Amer. Math. Soc. 2 (1989), no. 3, 599-635. MR991016 (90e:16049) 
18. G.A. Margulis, Explicit group-theoretic constructions of combinatorial schemes and their applications in the construction of expanders and concentrators, Problems of Information Transmission 24(1) (1988), 39-46. MR939574 (89f:68054)

19. J. Rogawski, Automorphic Representations of Unitary Groups in Three Variables, Princeton University Press, Princeton, NJ, 1990. MR.1081540 (91k:22037)

20. W. Scharlau, Zur Existenz von Involutionen auf einfachen Algebren. II, Math. Z. 176 (1981), 399-404. MR610220 (82k:12013)

21. A. Selberg, On discontinuous groups in higher-dimensional symmetric spaces, Contributions to function theory, International colloquia on function theory (Bombay, 1960), Tata Institute of Fundamental Research, Bombay, 1960, 147-164. MR0130324 (24:A188)

22. P. Solé, Ramanujan Hypergraphs and Ramanujan Geometries, Emerging Applications of Number Theory (eds. D.A. Hejhal, J. Friedman, M.C. Gutzwiller, A.M. Odlyzko), Springer, New York, 1999, 583-590. MR1691550 (2000c:05108)

23. J. Tits, Reductive groups over local fields, Automorphic forms, representations and $L$-functions (Proc. Sympos. Pure Math., Oregon State Univ., Corvallis, Ore., 1977), Part 1, 29-69, Proc. Sympos. Pure Math., XXXIII, Amer. Math. Soc., Providence, RI, 1979. MR546588 (80h:20064)

Department of Mathematics and Computer Science, College of the Holy Cross, WorCester, Massachusetts 01610

E-mail address: cballant@holycross.edu

Department of Mathematics, University of Utah, Salt Lake City, Utah 84112

E-mail address: ciubo@math.utah.edu 